Journal of Liquid Chromatography \& Related Technologies ${ }^{\circledR}$, 28: 289-295, 2005

Copyright (C) Taylor \& Francis, Inc.

ISSN 1082-6076 print/1520-572X online

DOI: $10.1081 / J L C-200041340$

\title{
Identification and Quantification of Bis-GMA and Teg-DMA Released from Dental Materials by HPLC
}

\author{
Marina Stefova and Violeta Ivanova \\ Institute of Chemistry, Faculty of Science, Skopje, Republic of \\ Macedonia \\ Ilijana Muratovska \\ Faculty of Dentistry, Skopje, Republic of Macedonia
}

\begin{abstract}
Most of the composites and sealants used in dentistry are based on bisphenol A diglycidylether methacrylate (Bis-GMA) and triethylenglycol dimethacrylate (Teg-DMA). Reports revealed that in situ polymerization is not complete and that free monomers can be detected by different analytical methods. We studied residual Bis-GMA and Teg-DMA after in vitro polymerization by high performance liquid chromatography (HPLC) after $24 \mathrm{hr}, 7$, and 14 days. For quantitative analysis, an $\mathrm{RP}$ column $(250 \times 4.6 \mathrm{~mm} ; 5 \mu \mathrm{m})$ as stationary phase and isocratic elution with $\mathrm{CH}_{3} \mathrm{CN}: \mathrm{H}_{2} \mathrm{O}(60: 40)$ was used. A calibration curve was constructed using standard solutions of Bis-GMA and Teg-DMA in the concentration range from $1 \times 10^{-3}$ to $5 \times 10^{-5} \mathrm{~mol} / \mathrm{L}$. The limits of detection (LOD) and quantification (LOQ) for both analytes were determined (for Bis-GMA: 0.0076 and $0.0229 \mathrm{mmol} / \mathrm{L}$; and for TegDMA: 0.0123 and $0.0373 \mathrm{mmol} / \mathrm{L}$, respectively). The accuracy of the method was confirmed by standard additions. The HPLC analysis of residual components revealed that Bis-GMA was released from commercial samples in the highest concentration after 14 days. Teg-DMA was detected only in one of all analyzed dental materials.
\end{abstract}

Keywords: Bis-GMA, TEG-DMA, Identification, Dental materials, HPLC

Address correspondence to Marina Stefova, Institute of Chemistry, Faculty of Science, P.O. Box 162, 1001, Skopje, Republic of Macedonia; E-mail: marinaiv@ iunona.pmf.ukim.edu.mk

Request Permissions / Order Reprints powered by $\frac{R \text { I G H T S L I N K }}{\text { S }}$ 


\section{INTRODUCTION}

Dental composite materials are today widely used not only because of their aesthetic properties, but for the ability to adhere to the tooth substance. Dental composite materials consist mainly of filler particles and a polymer matrix based on different monomers, dimmers, and/or oligomers of methacrylates and/or acrylates, together with additives. ${ }^{[1]}$

Polycarbonates, epoxy, and methacrylic resins are synthesized by reactions between individual chemicals, resulting in polymers with distinct physicochemical characteristics. ${ }^{[2]}$ As biomaterials they have multiple uses in human health applications; for instance, as manufactured products, for example, intraocular lenses, ${ }^{[3]}$ or cements for trauma surgery. ${ }^{[4,5]}$

The release of organic degradation products is dependent on the size of the molecules, where smaller molecules are presumed to have enhanced mobility and should, therefore, be eluted faster than larger molecules. ${ }^{[6,7]}$

Örtengren ${ }^{[1]}$ used high performance liquid chromatography (HPLC) for detection of Bis-GMA and Teg-DMA in the storage water. Quantifiable amounts of both substances were registered in the storage water for the studied materials. In the work of Pulgar, ${ }^{[2]}$ the oligomer, Bis-GMA leached from composites and sealants before and after polymerization was confirmed by HPLC analysis. Eight monomers extracted in five solvents were analyzed from different resins by Shintani ${ }^{[8]}$ by HPLC.

In this work, the leaching of bisphenol A diglycidylmethacrylate (Bis-GMA) and triethilenglycol dimethacrylate (Teg-DMA) was investigated. Residual monomers were determined using reversed phase HPLC (RP HPLC) with a UV diode array detector. The aim of our work was to identify and quantify Bis-GMA and Teg-DMA released from dental materials, through dentin in aqueous medium.

\section{EXPERIMENTAL}

\section{Reagents}

Acetonitrile (HPLC grade) from Aldrich was used. Reference standards were Bis-GMA (FW-512.21) and Teg-DMA (FW-286.148) kindly provided from Vivadent, Liechtenstein. Stock solutions of reference standards were prepared in ethanol.

\section{Dental Composites}

In this investigation, two different composite materials were employed: Tetric (Vivadent, Liechtenstein) and Esthet-X (Dentsply, De Tray). The basis of 
these composites consists of the monomers Bis-GMA and Teg-DMA. The recommended universal dentin-adhesive "Exite" (Vivadent, Liechtenstein) was used. Polymerization of the samples was performed by exposure to a halogen light using lamp "Astralis 7" (Vivadent, Liechtenstein).

\section{Sample Preparation}

Two groups of samples for each of the two commercial dental materials (Tetric and Esthet) were prepared with and without adhesive, polymerized for $20 \mathrm{sec}$ as abbreviated time of polymerization often used in practice, and for $40 \mathrm{sec}$ as recommended time of polymerization. The dental composites were deposited in a cavity (6 $\mathrm{mm}$ diameter and $2 \mathrm{~mm}$ deep) of extracted teeth from patients aged 18-25 years. In order to examine the leaching of monomers through dentin, these prepared teeth were placed in such a position to be in contact with water $(4 \mathrm{~mL})$ from the pulp side. After $24 \mathrm{hr}$, 7 , and 14 days, the water solutions were analyzed for detection and determination of the monomers Bis-GMA and Teg-DMA.

\section{HPLC Analysis}

The analysis of the monomers was carried out with a Varian HPLC system equipped with a ternary pump Model 9012 and UV Diode Array Detector Model 9065. The column used was C-18 Lichrospher 60 RP Select B $(250 \mathrm{~mm} \times 4 \mathrm{~mm}$, particle diameter $5 \mu \mathrm{m}$, Merck), and the mobile phase consisted of $\mathrm{CH}_{3} \mathrm{CN}$ and $\mathrm{H}_{2} \mathrm{O}(60: 40, \mathrm{~V} / \mathrm{V})$. The flow rate was $1 \mathrm{~mL} / \mathrm{min}$ and the sample volume: $20 \mu \mathrm{L}$. The elution was performed at room temperature and monitored in the whole UV range. For quantitation, the wavelength of $220 \mathrm{~nm}$ was used because both analytes exhibit significant absorption.

\section{RESULTS AND DISCUSSION}

Identification of the analytes Bis-GMA and Teg-DMA was made by comparison of the retention times and UV-spectra of the registered peak and the reference peak obtained for standards. The retention time obtained for Bis-GMA was $4.394 \mathrm{~min}$ and for Teg-DMA 6.401 min.

For quantitative analysis, a calibration curve was constructed using standard solutions of Bis-GMA and Teg-DMA in the concentration range from $1 \times 10^{-3}$ to $5 \times 10^{-5} \mathrm{~mol} / \mathrm{L}$. Detection was performed at the wavelength of $220 \mathrm{~nm}$ for both analytes because they exhibit significant absorption, which gives a good sensitivity of the method. The parameters obtained for 
the linear dependence peak area/concentration for the two analytes are the following:

$$
\begin{array}{ll}
\text { Bis-GMA } & A=1.2714 \times 10^{6} \times c+1.8198 \times 10^{4} \\
& R^{2}=0.9988 \quad \sigma=2.8007 \times 10^{4} \\
\text { Teg-DMA } & A=1.5127 \times 10^{6} \times c+3.9870 \times 10^{4} \\
& R^{2}=0.9992 \quad \sigma=2.7740 \times 10^{4}
\end{array}
$$

The limits of detection (LOD) and quantification (LOQ) were determined using calibration in the low concentration region $\left(5 \times 10^{-6}\right.$ to $\left.5 \times 10^{-5} \mathrm{~mol} / \mathrm{L}\right)$ as $3 \sigma /$ slope and $10 \sigma /$ slope, respectively, for both analyzed compounds, which is presented in Table 1.

The accuracy of the procedure was checked using the standard addition method. One real sample was spiked with appropriate volumes of a standard solution of Bis-GMA and satisfactory results for the recovery ranging from $98.61-104.03 \%$ (Table 2) confirmed that the method is accurate and convenient for quantitative analysis.

This procedure was then used for analysis of the residual monomers, which had penetrated in the water solution through the dental barrier after $24 \mathrm{hr}, 7$ and 14 days. Chromatograms of the analytes Bis-GMA and Teg-DMA obtained for samples and standard solution are given in Fig. 1.

After the 24th period, Bis-GMA was detected in all of the commercial samples, except Tetric without adhesive polymerized for $40 \mathrm{sec}$ and Esthet with adhesive also polymerized for $40 \mathrm{sec}$. After 7 days, Bis-GMA was found in all polymerized samples, except Esthet with adhesive polymerized for $40 \mathrm{sec}$. This substance was not detected in the Tetric sample without adhesive polymerized for $40 \mathrm{sec}$, the Esthet sample with adhesive polymerized for $40 \mathrm{sec}$, and Esthet sample without adhesive polymerized by exposure to light for $40 \mathrm{sec}$. In the Tetric sample with adhesive polymerized for $40 \mathrm{sec}$, Bis-GMA was detected in quantities close to LOD after $24 \mathrm{hr}$, and between LOD and LOQ after 7 and 14 days. The results for Bis-GMA are given in Table 3.

Table 1. Results obtained for LOD and LOQ for both analytes

\begin{tabular}{lccccc}
\hline Analyte & Regression equation & $R^{2}$ & $\sigma$ & $\begin{array}{c}\text { LOD } \\
(\mathrm{mmol} / \mathrm{L})\end{array}$ & $\begin{array}{c}\text { LOQ } \\
(\mathrm{mmol} / \mathrm{L})\end{array}$ \\
\hline Bis-GMA & $A^{a}=\begin{array}{c}1.2753 \times 10^{6} \times c \\
-2.9232 \times 10^{3}\end{array}$ & 0.9968 & 2921.81 & 0.0076 & 0.0229 \\
Teg-DMA & $\begin{array}{r}A=\begin{array}{l}1.7235 \times 10^{6} \times c \\
+1.6457 \times 10^{3}\end{array} \\
\hline\end{array}$ & 0.9928 & 6428.16 & 0.0123 & 0.0373 \\
\hline
\end{tabular}

${ }^{a} A$, peak area. 
Table 2. Results from the standard additions method for checking the accuracy of the HPLC method for determination of Bis-GMA in real samples $(n=3)$

\begin{tabular}{lccc}
\hline $\begin{array}{l}\text { Standard } \\
\text { addition }\end{array}$ & $\begin{array}{c}\text { Calculated, } \\
c(\mathrm{mmol} / \mathrm{L})\end{array}$ & $\begin{array}{c}\text { Experimentally } \\
\text { found, } c(\mathrm{mmol} / \mathrm{L})\end{array}$ & $\begin{array}{c}\text { Recovery } \\
(\%)\end{array}$ \\
\hline 1 & 0.0923 & 0.0961 & 104.03 \\
2 & 0.0885 & 0.0873 & 98.61 \\
3 & 0.0862 & 0.0856 & 99.28 \\
\hline
\end{tabular}

No detectable quantities of Teg-DMA were observed in Tetric and Esthet samples prepared with and without adhesive in that period, except in the sample of Esthet prepared without adhesive by exposure to light for $20 \mathrm{sec}$, in which $0.0777 \mathrm{mmol} / \mathrm{L}$ Teg-DMA were determined.

The HPLC analysis showed the highest concentration of monomers after 14 days in most samples. Ferrance ${ }^{[6]}$ has stated some factors of importance for the release of unbond substances from polymerized dental composites. He found that the monomer-polymer conversion determines the quantity of leachable components, because the kinetics and mechanism of elution processes depend on the composition and solubility parameters of the used solvent for extraction. The diffusion through the polymer network is determined by the size and chemical characteristics of the leachable substances.

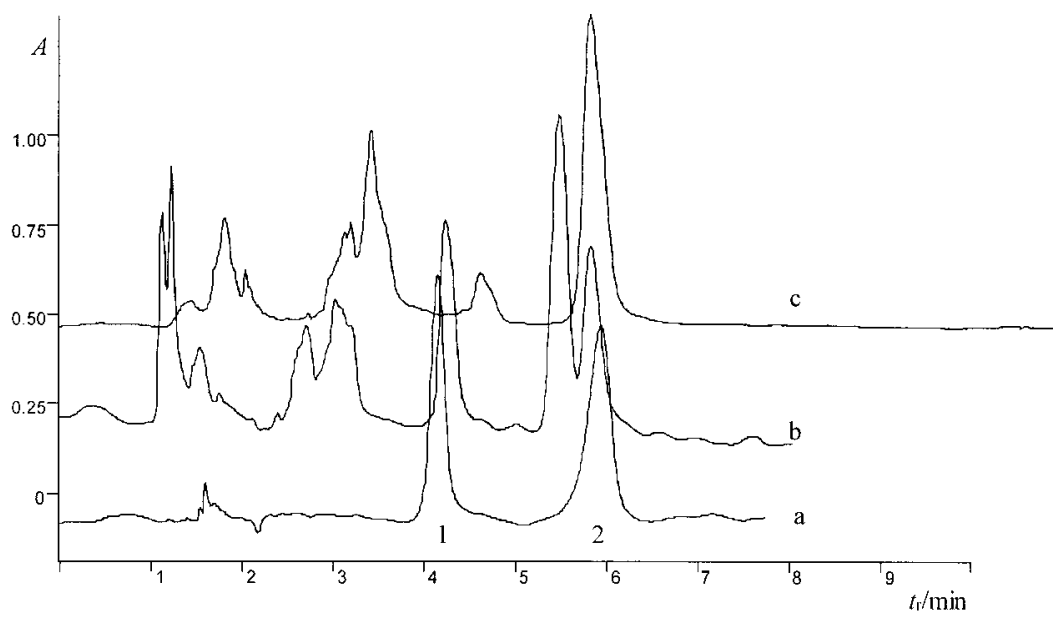

Figure 1. HPLC chromatograms of (a) a mixture of standard solutions of (1) Bis-GMA and (2) TEG-DMA, and samples of (b) Esthet with adhesive and (c) without adhesive polymerized by exposure to light for $20 \mathrm{sec}$ after 14 days. 
Table 3. Results from the determination of penetrated Bis-GMA in water through the dental barrier after $24 \mathrm{hr}, 7$ and 14 days in eight different samples of polymerized dental material

\begin{tabular}{|c|c|c|c|}
\hline Sample & $\begin{array}{c}c \text { (Bis-GMA) } \\
(\mathrm{mmol} / \mathrm{L}) \\
\text { after } 24 \mathrm{hr}\end{array}$ & $\begin{array}{c}c(\text { Bis-GMA) } \\
(\mathrm{mmol} / \mathrm{L}), \\
\text { after } 7 \text { days }\end{array}$ & $\begin{array}{c}c \text { (Bis-GMA) } \\
(\mathrm{mmol} / \mathrm{L}) \\
\text { after } 14 \text { days }\end{array}$ \\
\hline Tetric $20 \sec ^{a}$ with adhesive & 0.0358 & 0.0847 & 0.0975 \\
\hline Tetric $40 \sec ^{a}$ with adhesive & $\sim$ LOD & $\begin{array}{l}\text { Between LOD } \\
\text { and LOQ }\end{array}$ & $\begin{array}{l}\text { Between LOD } \\
\text { and LOQ }\end{array}$ \\
\hline Tetric $20 \mathrm{sec}$ without adhesive & $\sim \mathrm{LOD}$ & $\sim \mathrm{LOD}$ & $\sim \mathrm{LOD}$ \\
\hline Tetric $40 \mathrm{sec}$ without adhesive & - & 0.0768 & - \\
\hline Esthet $20 \mathrm{sec}$ with adhesive & 0.1394 & 0.2598 & 0.2708 \\
\hline Esthet $40 \mathrm{sec}$ with adhesive & - & - & - \\
\hline Esthet $20 \mathrm{sec}$ without adhesive & $\begin{array}{c}\text { Between LOD } \\
\text { and LOQ }\end{array}$ & 0.0451 & 0.0760 \\
\hline Esthet $40 \mathrm{sec}$ without adhesive & 0.0269 & 0.0344 & - \\
\hline
\end{tabular}

$a_{20}$ and $40 \mathrm{sec}$ is the time of exposure to halogen light.

It is shown that the composites and sealants are unstable and that to a greater or lesser degree, depending on the aggressiveness of the medium, it is always possible to detect the elution of monomers, olygomers, and precursors. These findings confirm the reports that in vivo polymerization is not complete and that free monomers can be detected by different analytical methods. ${ }^{[8-11]}$ In this work, leaching of residual monomers Bis-GMA and Teg-DMA in water through dentin was measured employing polymerization by exposure to light for 20 and $40 \mathrm{sec}$. The higher quantities of monomers obtained for reduced exposure to light show that the recommended exposure time $(40 \mathrm{sec})$ should be used. Also, lower results for residual monomers obtained for samples when an adhesive is used recommend employing adhesives, together with the suggested exposure time for complete polymerization.

The proposed HPLC method for analysis of residual monomers in polymerized dental composites is simple, rapid, and sensitive. It is suitable for identification and quantification of the monomers Bis-GMA and Teg-DMA from various samples.

\section{REFERENCES}

1. Örtenger, U.; Wellendorf, H.; Karlson, S.; Ruiter, I.E. Water sorption and solubility of dental composites and identification of monomers released in an aqueous environment. J. Oral Rehabil. 2001, 28, 1106-1115. 
2. Pulgar, R.; Olea-Serrano, M.F.; Novillo-Fertrell, A.; Rivas, A.; Pazos, P.; Pedraza, V.; Navajas, J.M.; Olea, N. Determination of bisphenol A and related aromatic compounds released from Bis-GMA-based composites and sealants by high performance liquid chromatography. Environ. Health Perspect. 2000, 108, 1.

3. Yalon, M.; Goldberg, E.P.; Osborn, D.; Stacholy, J.; Sheets, J.W. Polycarbonate intraocular lenses. J. Cataract Refract. Surg. 1988, 1449, 393-395.

4. Vuillemin, T.; Raveh, J.; Stich, H.; Cottier, H. Fixation of bone fragments with BIOCEM. First observations on humans. Arch. Otolaryngol. Head Neck Surg. 1987, 113, 836-840.

5. Kanawabe, K.; Tamura, J.; Yamamuro, T.; Nakamura, T.; Kokubo, T.; Yoshihara, S. A new bioactive bone cement consisting of Bis-GMA resin and bioactive glass power. J. Appl. Biomat. 1995, 4, 135-141.

6. Ferracane, J.L. Elution of leachable components from composites. J. Oral Rehabil. 1994, $21,441$.

7. Geurtzen, W. Substances released from dental resin composites and glass ionomer cements. Eur. J. Oral Sci. 1998, 106, 687.

8. Shintani, H. HPLC analysis of toxic additives and residual monomer from dental plate. J. Liq. Chromatogr. 1995, 18, 613-626.

9. Hamid, A.; Hume, W.R. A study component release from resin pit and fissure sealants in vitro. Dent. Mater. 1997, 13, 98-102.

10. Nathanon, D.; Lertpitayakun, P.; Lamkin, M.S.; Mahnaz, E.B.; Lee-Chou, L. In vitro elution of leachable components from dental sealants. J. Am. Dent. Assoc. 1997, 128, 1517-1523.

11. Rueggeberg, F.A.; Craig, R.G. Correlation of parameters used to estimate monomer conversion in a light cured composite. J. Dent. Res. 1988, 67, 932-937.

Received August 1, 2004

Accepted August 20, 2004

Manuscript 6473 